\title{
BER Performance of 1024 (BPSK, QPSK \& QAM) using OFDM over Rayleigh, Rician and Nakagami Fading Channel
}

\author{
P. Rajasekhar \\ P.G. Scholar, Vishnu \\ Institute of Technology, \\ Vishnupur, Bhimvaram
}

\author{
V. Rama Raju \\ Associate Professor, \\ Dept. Of ECE, Vishnu \\ Institute of Technology, \\ Vishnupur, Bhimvaram
}

\begin{abstract}
To enhance the band width usage with adding cyclic prefix and pilot insertion, wavelet based OFDM is employed for performance increment using BER analysis. This also classified on AWGN, RICIAN, RAYLEIGH and NAKAGAMI channels for BER performance based on SNR. These results as numerically compared with original OFDM, FRFT-OFDM
\end{abstract}

\section{Keywords}

Band Width, cyclic prefix, pilot insertion, wavelet, OFDM, BER, AWGN, RICIAN, RAYLEIGH, NAKAGAMI, SNR, FRFT-OFDM, DWT-FRFT-OFDM.

\section{INTRODUCTION}

The desire for faster wireless technologies and the increase in multimedia applications is the principal driving force behind OFDM's increased popularity. Orthogonal Frequency Division Multiplexing (OFDM) is a modulation scheme used to transmit digital data efficiently in multipath fading environment. It is a multi-carrier system with simultaneous transmission of data on closely spaced orthogonal subcarriers. Conventional OFDM system is deployed with IFFT (Inverse Fourier Transform) to generate orthogonal subcarriers. A cyclic prefix is added to every symbol to combat the delay spread introduced by multipath channel and the length of cyclic prefix must be at least the same size as the expected channel delay spread. Addition of cyclic prefix minimizes inter-symbolinterference (ISI) [1].

With the rapid growth of digital communication in recent years, the need for high-speed data transmission has been increased. The wireless industry faces the problem of providing the technology that be able to support a variety of services ranging from voice communication with a bit rate of a few kbps to wireless multimedia in with a bit rate up to 2 Mbps. Many systems have been proposed and OFDM system has gained much attention for different reasons. Although OFDM was first proposed in the 1960s, only in recent years, it has been recognized as an outstanding method for high-speed data communication system where its implementation relies on very high-speed digital signal processing. Since OFDM is carried out in the digital domain, OFDM method is flexible for the design process and enough fast in terms of time to put it in the market [2].

OFDM is a multicarrier modulation technique. OFDM provides high bandwidth efficiency because the carriers are orthogonal to each other and multiple carriers share the data among themselves. The main advantage of this transmission technique is their robustness to channel fading in wireless communication environment. The main objective of this project is to design a simulation model using MATLAB to check the performance of the paper design [3].

Orthogonal Frequency Division Multiplexing (OFDM) is a multi-carrier modulation technique which divides the available spectrum into many carriers. OFDM uses the spectrum efficiently compared to FDMA by spacing the channels much closer together and making all carriers orthogonal to one another to prevent interference between the closely spaced carriers. The main advantage of OFDM is their robustness to channel fading in wireless environment. Commercial applications of OFDM are ADSL, IEEE802.11a/HiperLAN2, WiMAX, Digital Broadcasting (DVB-T) [4].

\section{METHODOLOGY}

Block diagram representation is provided below with mathematical representation

\section{A. FRFT}

The meaning of FrFT states it as a chrip basis extension, which is characterizing the revolution in time, frequency that is brought together time frequency transformation by changing the estimation of Fractional. By changing the Fractional estimation from 0.0 to 1.0 the signal characterstics can be transformed from time to frequency domain[5].

FrFT is defined as

$$
F_{\alpha}\{x(t)\}(u)=\int_{-\infty}^{\infty} x(t) k_{\alpha}(t, u) d t
$$

$\mathrm{K} \alpha(t, u)=A_{\alpha} e^{j \pi\left(t^{2}+u^{2}\right) \cot \alpha-j 2 \pi t u c s c \alpha} \quad$ is called the kernel transform where $\alpha$ is called the rotation angle of a transformed signal and $\alpha=\frac{a \pi}{2}[6]$.By estimating the fractional component ' $a$ ' improvement in BER can be achieved. FrFT appended OFDM signal must be orthogonal as to achieve error free signal For orthogonality condition between any two signals we must compute the whole interval i.e(-T/2 to $+\mathrm{T} / 2)$ Consider two signals $X_{\alpha, m}(t)$ be the FrFt appended OFDM signal and $X_{\alpha, n}^{*}(\mathrm{t})$ be its orthogonal basis where $\mathrm{m}, \mathrm{n}$ are called the subcarriers

Then from[7]

$$
\begin{aligned}
& \int_{-T / 2}^{+T / 2} X_{\alpha, m}(t) \cdot X_{\alpha, n}^{*}(t) d t \\
& =\frac{1}{2 \pi} e^{j \frac{\left(\left(n t_{0}\right)^{2}-\left(m t_{0}\right)^{2}\right) \cot \alpha}{2}} \int_{-T / 2}^{+T / 2} e^{j(m-n) t_{0} t c s c \alpha} d t(2)
\end{aligned}
$$




\section{Where $t_{0}$ is the central frequency}

\section{FrFT appended OFDM}

FrFT is used to detect and estimate the interference component

The original OFDM modulated signal was given by

$x(t)=\sum_{n=1}^{N} m_{n}(t) \sin (2 \pi n t)$

The above signal was passed through FrFT represented by $F^{+\alpha}$ and the signal is passed through AWGN, Rayleigh, Racian, Nakagami

After passing through FrFT the signal is given by

$\left\{F^{+\alpha}\{x(t)\}\right\}=\sqrt{\frac{1-i \cot \theta}{2 \pi}} \int_{-\frac{T}{2}}^{+\frac{T}{2}} x(t) e^{\frac{j}{2}\left(u^{2}+t^{2}\right)^{\cot \theta-i u t c s c \theta}}$

By keeping the value of $\mathrm{x}(\mathrm{t})$ from $\mathrm{Eq}(3)$ and $\mathrm{Eq}(4)$

Becomes

$$
\left\{F^{+\alpha}\{x(t)\}\right\}=\sqrt{\frac{1-i \cot \theta}{2 \pi}} \times A
$$

Where

$$
A=\int_{+T / 2}^{-T / 2} \sum_{n=1}^{N} m_{n}(t) \sin (2 \pi n t) \cdot e^{\frac{j}{2}\left(u^{2}+t^{2}\right)^{\cot \theta-i u t c s c \theta}}
$$

(5)

This $\mathrm{Eq}(5) \mathrm{FrFT}$ is transmitted through different wireless channels

After the channel the signal is passed through after which the signal $X_{\alpha}$ is passed through Inverse Fractional Fourier Transform(IFrFT) given by

$$
\begin{aligned}
& \left\{F^{-\alpha}\left\{F^{+\alpha}\{x(t)\}\right\}\right\}=F^{-\alpha}\left\{\sqrt{\frac{1-i \cot \theta}{2 \pi}} \times A\right\} \\
& \left\{F^{-\alpha}\left\{F^{+\alpha}\{x(t)\}\right\}\right\}=x(t)
\end{aligned}
$$

The original transmitted signal is recovered after passing through IFrFT when fractional value $\mathrm{a}=1$ the conventional Fourier transform is obtained

\section{B. Noise channels}

Additional White Gaussian Noise (AWGN) channel the received signal is equal to the transmitted signal with some portion of white Gaussian white noise added. This channel is particularly important for discrete models operating on a restricted number space, because this allows one to optimise the circuits in terms of their noise performance.

$$
s(t)=A+n(t)(7)
$$

Rayleigh fading is caused by multipath reception. The mobile antenna receives a large number, say $N$, reflected and scattered waves. Because of wave cancellation effects, the instantaneous received power seen by a moving antenna becomes a random variable, dependent on the location of the antenna. To simplify the derivation of the fading models an un-modulated carrier of the form as transmission signal is used.

$$
s(t)=A \sum_{i=0}^{D(t)-1} a_{i}(t) \cdot \cos \left\{2 \pi f\left[t-\tau_{i}(t)\right]\right\}(8)
$$

Rician fading channel indicates that there is a prominent or direct path over which the electromagnetic wave can travel. Compared to the Rayleigh channel model, Equation 1, the Rician fading channel model has an additional component to reflect the prominent path.

$$
s(t)=A \cdot \cos (2 \pi f t)+\sum_{i=0}^{D(t)-1} a_{i}(t) \cdot \cos \left\{2 \pi f\left[t-\tau_{i}(t)\right]\right\}(9)
$$

Nakagami fading model was initially proposed because it matched empirical results for short wave ionospheric propagation. In current wireless communication, the main role of the Nakagami model can be summarized as follows

- It describes the amplitude of received signal after maximum ratio diversity combining.

- The sum of multiple independent and identically distributed (i.i.d.) Rayleigh-fading signals have a Nakagami distributed signal amplitude. This is particularly relevant to model interference from multiple sources.

- The Nakagami distribution matches some empirical data better than other models

- The Rician and the Nakagami model behave approximately equivalently near their mean value. This observation has been used in many recent papers to advocate the Nakagami model as an approximation for situations where a Rician model would be more appropriate.

For Nakagami fading, the instantaneous power has the gamma pdf

$$
f_{p_{i}}\left(p_{i}\right)=\frac{1}{G(m)}\left(\frac{m}{\overline{p_{i}}}\right)^{m} p_{i}^{m-1} e^{\left\{-\frac{m p_{i}}{p_{i}}\right\}}(10)
$$

where $\mathrm{G}(m)$ is the gamma function, with $\mathrm{G}(m+1)=m$ ! for integer shape factors $m$. The mean value is $p_{i}$. In the special case that $m=1$, Rayleigh fading is recovered, while for larger $m$ the spread of the signal strength is less, and the pdf converges to a delta function for increasing $m$. 


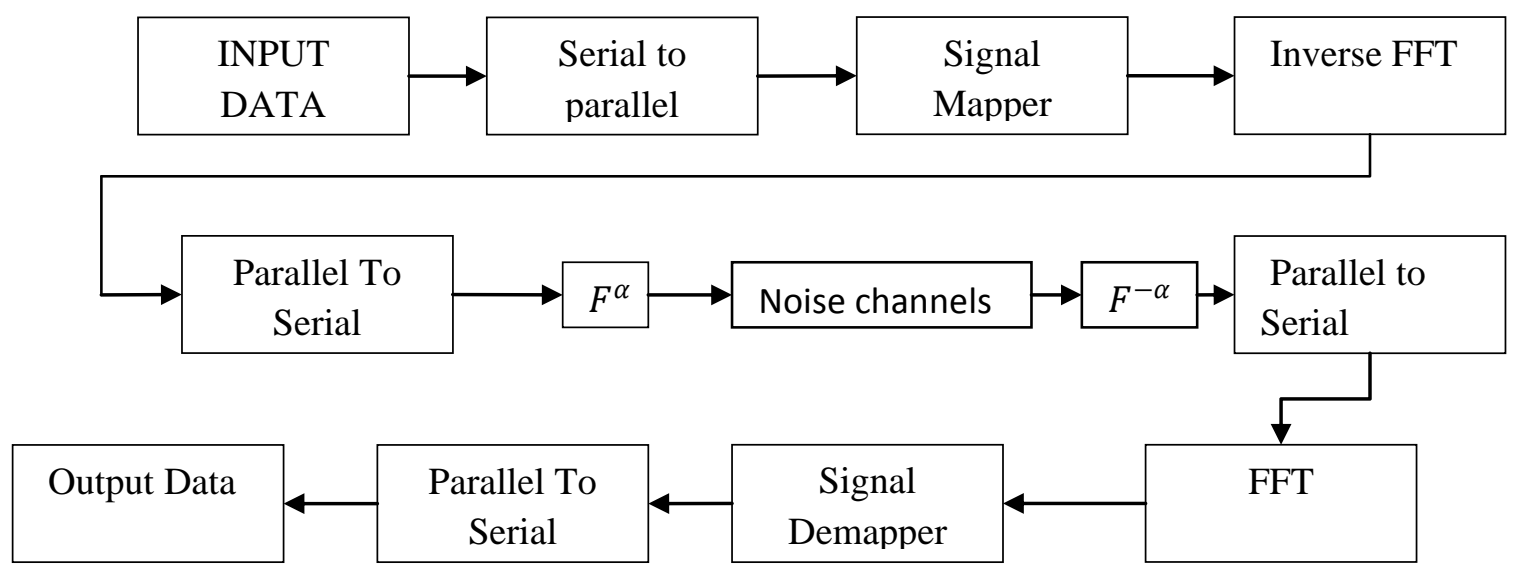

Figure 5 Block Diagram of Proposing Scheme

Table 1: BPSK Modulated FrFT OFDM @ Normalisation factor $\mathbf{a}=0.3$ and $\mathbf{a}=1$.

\begin{tabular}{|c|c|c|c|c|c|c|}
\hline \multicolumn{7}{|c|}{ For BPSK } \\
\hline \multirow[t]{2}{*}{ S.No } & \multirow[t]{2}{*}{ Channels } & \multirow[t]{2}{*}{ BER(in dB) } & \multirow[t]{2}{*}{$\begin{array}{l}\text { Original } \\
\text { System(SNR) }\end{array}$} & \multicolumn{2}{|c|}{$\begin{array}{l}\text { Proposed system(SNR) } \\
\text { frft_OFDM }\end{array}$} & \multirow[t]{2}{*}{$\begin{array}{l}\text { Improvement } \\
\text { in SNR }\end{array}$} \\
\hline & & & & $a_{o p t}$ & SNR & \\
\hline 1. & AWGN & $10^{-2.1}$ & 5 & 0.3 & 20 & 15 \\
\hline 2. & RAYLEIGH & $10^{-2.4}$ & 24.3 & 0.3 & 25 & 0.7 \\
\hline 3. & RICIAN & $10^{-2.9}$ & 34 & 0.3 & 22.3 & 11.5 \\
\hline 4. & NAKAGAMI & $10^{-3.1}$ & 18 & 0.3 & 30 & 2 \\
\hline
\end{tabular}

Table 2: M PSK Modulated FrFT OFDM @ Normalisation factor $\mathrm{a}=\mathbf{0 . 3}$ and $\mathrm{a}=1$.

\begin{tabular}{|l|l|l|l|l|l|l|}
\hline \multicolumn{2}{|l|}{ For MPSK } & BER(in dB) & $\begin{array}{l}\text { Original } \\
\text { System(SNR) }\end{array}$ & $\begin{array}{l}\text { Proposed system(SNR) } \\
\text { frft_OFDM }\end{array}$ & $\begin{array}{l}\text { Improvement } \\
\text { in SNR }\end{array}$ \\
\hline S.No & Channels & & & SNR & \\
\cline { 5 - 8 } & & & & $\mathrm{a}_{\text {opt }}$ & 30 & 19 \\
\hline 2. & RAYLEIGH & $10^{-2.4}$ & 34.5 & 0.3 & 35 & 0.5 \\
\hline 3. & RICIAN & $10^{-2.9}$ & 42 & 0.3 & 43.5 & 1.5 \\
\hline 4. & NAKAGAMI & $10^{-3.1}$ & 28 & 0.3 & 29 & 1 \\
\hline
\end{tabular}

Table 3: MQAM Modulated FrFT OFDM @ Normalisation factor a = 0.3 and a=1.

\begin{tabular}{|c|c|c|c|c|c|c|}
\hline \multicolumn{7}{|c|}{ M-QAM } \\
\hline \multirow[t]{2}{*}{ S.No } & \multirow[t]{2}{*}{ Channels } & \multirow[t]{2}{*}{$\mathrm{BER}($ in $\mathrm{dB})$} & \multirow{2}{*}{$\begin{array}{l}\text { Original } \\
\text { System(SNR) }\end{array}$} & \multicolumn{2}{|c|}{ Proposed system } & \multirow{2}{*}{$\begin{array}{l}\text { Improvement } \\
\text { in SNR }\end{array}$} \\
\hline & & & & $a_{o p t}$ & SNR & \\
\hline 1. & AWGN & $10^{-2.1}$ & 25 & 0.3 & 28 & 3 \\
\hline 2. & RAYLEIGH & $10^{-2.4}$ & 55 & 0.3 & 50 & 5 \\
\hline 3. & RICIAN & $10^{-2.9}$ & 56 & 0.3 & 57.5 & 1.5 \\
\hline 4. & NAKAGAMI & $10^{-3.1}$ & 43 & 0.3 & 45 & 2 \\
\hline
\end{tabular}




\section{RESULTS}

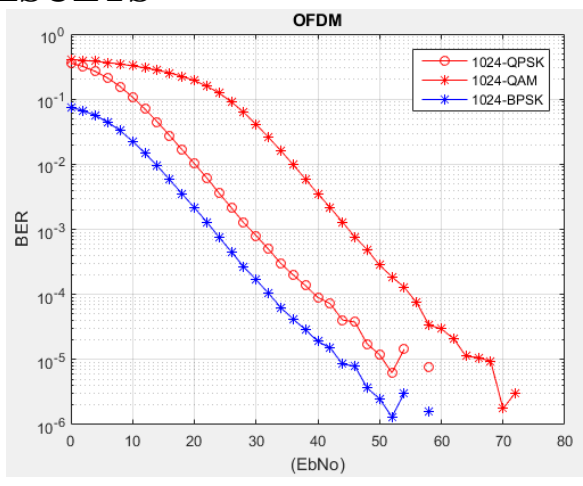

Figure 6 Awgn Channel

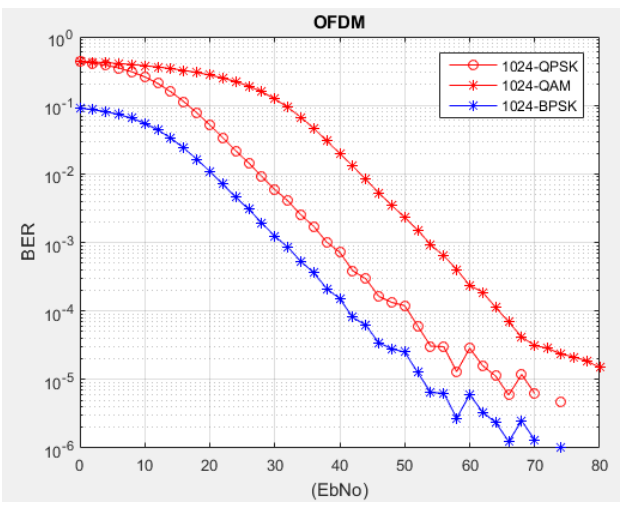

Figure 7 Rayleigh FRFT Channel

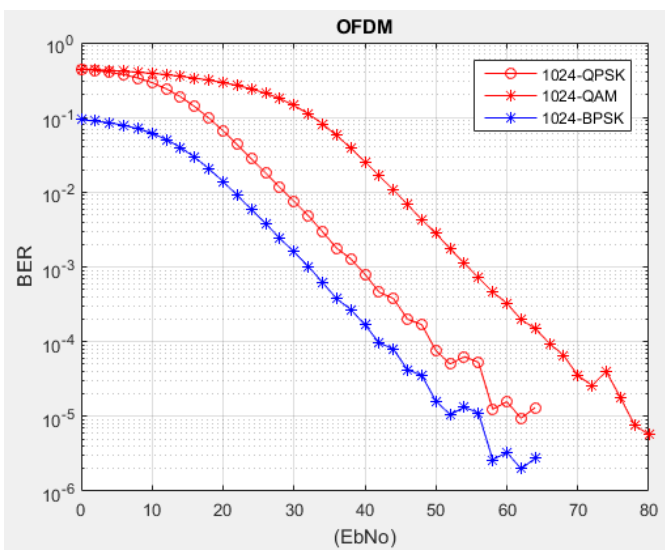

Figure 8rician FRFT Channel

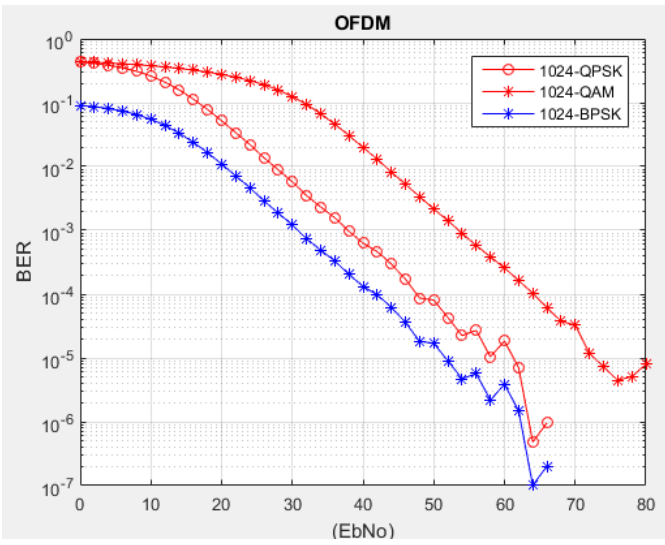

Figure 7 Nakagami FRFT Channel

\section{CONCLUSION}

Obtaining best results using BPSK Modulated signal using FRFT OFDM which helps in achieving maximised BER performance under different noise channels. Here at maximum SNR the best BER are tabulated. From that we see for Rayleigh channel $10^{-2.4}$ BER at 20dB SNR is achieved for 1024 QPSK and for racian channel $10^{-2.9} \mathrm{BER}$ at $25 \mathrm{~dB}$ SNR is achieved for 1024 QAM and for Nakagami channel $10^{-3.1}$ BER at 30dB SNR is achieved for 1024 QAM and for the AWGN channel $10^{-2.1}$ BER at $22.3 \mathrm{~dB}$ SNR is achieved for 1024 QPSK modulation.

\section{FUTURESCOPE}

Encryption based DWT-FrFT will leads to securable communication process.

\section{REFERENCES}

[1]Mrs.Veena M.B. Dr.M.N.ShanmukhaSwamy,"Performance analysis of DWT based OFDM over FFT based OFDM and implementing on FPGA" International Journal of VLSI design \& Communication Systems (VLSICS) Vol.2, No.3, September 2011.

[2] Deepak Gupta, Vipin B Vats, Kamal K. Garg, "Performance Analysis of DFT-OFDM, DCT-OFDM and DWT-OFDM Systems in AWGN Channel" International Conference on Wireless and Mobile Communications ICWMC'08, July 27 - Aug 01, 2008.

[3] Swati Sharma, Sanjeevkumar, "BER Performance Evaluation of FFT-OFDM and DWTOFDM"International Journal of Network and MobileTechnologiesISSN 2229-9114 Electronic VersionVOL 2 / ISSUE 2 / MAY 2011.

[4] LalchandraPatidar, Ashish Parikh, "BER Comparison of DCT-based OFDM and FFT-based OFDM using BPSK Modulation over AWGN and Multipath Rayleigh Fading Channel" International Journal of Computer Applications (0975 - 8887) Volume 31- No.10, October 2011.

[6] R. Saxena, K. Singh, Fractional Fourier transform: a novel tool for signalprocessing, Indian Inst. Sci. 85 (2005) 1126

[8]N. Prasad, S. Wang, N. Wang, Efficient receiver algorithms for DFT-spread OFDMsystems, IEEE Trans. Wirel. Commun. 8 (2009) 3216-3225

[9] Stefano Galli, O. Logvinov, "Recent Developments in the Standardization of Power Line Communications within the IEEE". IEEE Communications Magazine. 46 (7): 64 71 , July 2008 .

[10] StéphaneMallat, A Wavelet Tour of Signal Processing, Academic Press, 1999.

[11] C. S. Burrus, R. A. Gopinath, H. Guo, Introduction to Wavelets and Wavelet Transforms, a primer, Prentice Hall New jersey, 1998. 